\title{
Medición de TSH, T4 y Phe en muestras de sangre del cordón umbilical en papel de filtro: impacto en el tamizaje neonatal
}

\author{
Neivis Marrero ${ }^{1}$, Amarilys Frómeta ${ }^{1}$, Remigio Coto ${ }^{2}$, Layda Villegas ${ }^{2}$ \\ ${ }^{1}$ Laboratorio de Tamizaje Neonatal, Centro de Inmunoensayo, La Habana, Cuba \\ ${ }^{2}$ Laboratorio SUMA, Hospital Docente Materno-Infantil 10 de octubre, La Habana, Cuba
}

\begin{abstract}
Resumen
En este momento existe consenso en que el hipotiroidismo congénito y la fenilcetonuria constituyen modelos teóricos y prácticos ideales en el tamizaje neonatal. La tendencia actual en el mundo es la salida temprana de las maternidades debido a razones socioeconómicas, lo cual guía, sin duda, a la implementación de estos programas en sangre del cordón umbilical. Se evaluaron los niveles de T4 total, TSH y Phe en muestras de sangre del cordón umbilical de 458 recién nacidos, colectadas en papel de filtro S\&S 2992, utilizando ultramicroensayos fluorescentes. La distribución de frecuencias para las concentraciones de TSH mostró un valor medio de $1,7 \mathrm{mUI} / \mathrm{l}$; el $98 \%$ de las muestras tuvieron concentraciones en el rango de 0-10 $\mathrm{mUI} / \mathrm{l}$, el $1,7 \%$ entre $10-20 \mathrm{mUI} / \mathrm{l}$ y una muestra con valor mayor de $20 \mathrm{mUl} / \mathrm{l}$; para T4 el valor medio de concentración fue de $177,2 \mathrm{nmol} / \mathrm{l}$; el $90,2 \%$ tuvo valores entre $116-370 \mathrm{nmol} / \mathrm{l}$, el $9,6 \%$ entre $60-116 \mathrm{nmol} / \mathrm{l}$ y una muestra con un valor menor de $60 \mathrm{nmol} / \mathrm{l}$. Para Phe, el valor promedio fue de 4,6 $\mu \mathrm{mol} / / \mathrm{l}(0,7 \mathrm{mg} / \mathrm{dl})$, el 80,3\% entre $0-60 \mu \mathrm{mol} / \mathrm{l}(0-1 \mathrm{mg} / \mathrm{dl})$, el $19,2 \%$ entre $60-120 \mu \mathrm{mol} / \mathrm{l}$ (1-2 mg/dl) y 2 muestras con valor mayor de $120 \mu \mathrm{mol} / \mathrm{l}(2 \mathrm{mg} / \mathrm{dl})$. Los resultados obtenidos demuestran que es factible el empleo de muestras en sangre del cordón para el diagnóstico precoz de hipotiroidismo congénito, no así en el caso de la Phe donde los niveles detectados fueron muy bajos por lo que no garantiza total efectividad en el diagnóstico de fenilcetonuria.
\end{abstract}

Palabras clave: hipotiroidismo congénito, fenilcetonuria (PKU), tamizaje neonatal, fenilalanina (Phe), hormona estimulante de la tiroides (TSH), tiroxina (T4).

Measurements of TSH, T4 and Phe in samples of umbilical cord blood on filter paper: impact on neonatal screening

\begin{abstract}
There is a consensus on considering congenital hypothyroidism and phenylketonuria as theoretical and practical models in neonatal screening. The tendency toward early discharge in some maternity hospitals due to socioeconomic reasons led us to implement screening programs in umbilical cord blood samples. Titres of total T4, TSH and Phe in cord blood on S\&S 2992 filter paper from 458 neonates were performed using fluorescent ultramicroassays. Frequency distribution for TSH concentrations showed a mean of 1.7 $\mathrm{mIU} / \mathrm{l} ; 98 \%$ of the samples had concentrations within the $0-10 \mathrm{mIU} / \mathrm{l}$ range, $1.7 \%$ within $10-20 \mathrm{mIU} / \mathrm{l}$ and one sample with a value higher than $20 \mathrm{mIU} / \mathrm{l}$. The mean value was 177.2 $\mathrm{nmol} / \mathrm{l}$ for T4, $90.2 \%$ within $116-370 \mathrm{nmol} / \mathrm{l}, 9.6 \%$ within $60-116 \mathrm{nmol} / \mathrm{l}$ and one sample with a value lower than $60 \mathrm{nmol} / \mathrm{l}$. For Phe, the mean concentration was $41.6 \mu \mathrm{mol} / \mathrm{l}(0.7$ $\mathrm{mg} / \mathrm{dl}), 80.3 \%$ within the range $0-60 \mu \mathrm{mol} / \mathrm{l}(0-1 \mathrm{mg} / \mathrm{dl}), 19.2 \%$ within $60-120 \mu \mathrm{mol} / \mathrm{l}(1-2$ $\mathrm{mg} / \mathrm{dl})$ and two samples with a value higher than $120 \mu \mathrm{mol} / \mathrm{l}(2 \mathrm{mg} / \mathrm{dl})$. Results showed
\end{abstract}


that the use of cord blood samples in early diagnosis of congenital hypothyroidism is convenient. However, cord blood Phe levels were lower. Thus, they do not guarantee total effectiveness in the detection of phenylketonuria.

Key words: congenital hypothyroidism, phenylketonuria (PKU), neonatal screening, phenylalanine (Phe), thyroid stimulating hormone (TSH), thyroxine (T4).

El tamizaje clásico en una población de recién nacidos se lleva a cabo para enfermedades clínicamente no detectables y que sean tratables antes de que los efectos sean irreversibles. Los errores congénitos han constituido la mayor causa de patología neonatal, aunque han disminuido notablemente por los avances en las conductas obstétricas prenatales y perinatales.

Los programas de detección temprana de enfermedades metabólicas en recién nacidos constituyen un acto de medicina preventiva en el marco de la salud pública, para la identificación precoz de enfermedades que pueden conducir potencialmente a problemas de graves consecuencias para la salud y la sociedad. Ellos exploran los riesgos individuales de una población dada y una vez encontrados, se requiere de exámenes confirmatorios individuales y del diagnóstico.

Los programas de tamizaje neonatal comenzaron en EUA en 1961, con la determinación de Phe en sangre seca sobre papel de filtro con la prueba de inhibición bacteriana de Guthrie, para la detección de fenilcetonuria (1). Una década después, en 1972, se inició en Quebec un programa para la detección de hipotiroidismo congénito (2), seguido por el de Pittsburgh en 1973 (3).

En este momento, existe consenso en que estas enfermedades constituyen modelos teóricos y prácticos ideales en el tamizaje neonatal para enfermedades metabólicas hereditarias; han sido los programas de tamizaje más extendidos en el mundo, contando hoy con una experiencia de casi tres décadas de funcionamiento.

Correspondencia: Neivis Marrero, Calle 134 y Avenida 25, Apartado postal 6653, Cubanacán, Playa, Ciudad de La Habana, Cuba

Recibido: 30/08/99; aceptado: 11/02/00
La fenilcetonuria es un desorden congénito del metabolismo de la Phe, con una frecuencia de 1:16.000 recién nacidos (4), producida por la disminución o deficiencia de la actividad del complejo enzimático hidroxilasa de fenilalanina, lo que provoca la acumulación de Phe en sangre y orina que, a su vez, induce un grave retardo mental (5). Por esta razón, es imprescindible la detección temprana y la aplicación de una dieta deficiente de Phe, con cantidad suficiente de este aminoácido para garantizar el desarrollo normal del niño (6).

El hipotiroidismo congénito es uno de los desórdenes endocrinos más frecuentes en la infancia (7). Esta enfermedad es causada por la ausencia anatómica o funcional de la glándula tiroides y constituye la causa más frecuente de retraso mental evitable, con una frecuencia aproximada de 1:4.000 recién nacidos $(8,9)$; sólo el diagnóstico precoz y una terapia sustitutiva con L-tiroxina en los primeros momentos de la vida pueden prevenir las consecuencias adversas en el desarrollo psicomotor (10-14).

Uno de los problemas a los que se enfrentan las autoridades de salud al ejecutar los programas de tamizaje neonatal es lograr una alta cobertura, para lo cual es necesario tener en cuenta las estructuras de salud de cada país, el porcentaje de partos que ocurre en centros hospitalarios, y la sensibilización y educación de los padres.

En Latinoamérica, algunos países como Chile, Costa Rica, Puerto Rico, Uruguay y Cuba cuentan con un programa nacional con cobertura cercana al $100 \%$ de los recién nacidos. En Brasil y Argentina, el diagnóstico neonatal se realiza en algunas provincias con excelente cobertura y con la posibilidad de ampliarla (15).

Los porcentajes de cobertura son variables: Cuba, 98\%; Chile, 96,5\%; Uruguay, 99\%; Costa Rica, 
91\%; Brasil, $60 \%$; Argentina, 44,5\%, y México, $38,6 \%$ (16).

La toma de muestra de sangre del talón del recién nacido en papel de filtro entre el segundo y el séptimo dia de nacido, se ha extendido en la mayoría de estos programas de tamizaje neonatal. Sin embargo, hay países como Cuba y Uruguay, donde se ha establecido el uso de muestras del cordón umbilical como una estrategia válida para lograr un óptimo desarrollo de sus programas $(17,18)$

La tendencia actual en el mundo es la salida temprana de las maternidades, lo cual conlleva, sin duda, a la implementación de programas de tamizaje en sangre del cordón con el objetivo de aumentar el porcentaje de cobertura de dichos programas $(19,20)$.

Este trabajo pretende analizar los niveles de T4 total, TSH y Phe en sangre del cordón umbilical y valorar la factibilidad de su uso como marcadores para el diagnóstico temprano de hipotiroidismo congénito y fenilcetonuria, respectivamente.

\section{Materiales y métodos}

\section{Muestras de sangre}

Las muestras usadas en este estudio se obtuvieron de sangre del cordón umbilical, para lo cual se depositaron $40 \mu \mathrm{l}$ ( 2 gotas) de EDTA al $4 \%$ en un tubo de ensayo, se despinzó suavemente el extremo del cordón umbilical próximo a la placenta y se dejó drenar espontáneamente la sangre en el tubo; se recolectaron $2 \mathrm{ml}$ de sangre y se homogenizó suavemente. Se dispensaron $100 \mu$ l de sangre (con pipeta automática de volumen fijo o gotero desechable, tarado para este volumen), en cada uno de los 4 círculos impresos en las tarjetas de papel de filtro Schleicher y Schuell 2992 y se colocaron en posición horizontal sobre un soporte diseñado al efecto; se dejaron secar durante 3 horas a temperatura ambiente y, luego, se guardaron en sobres de papel y se conservaron a $-20^{\circ} \mathrm{C}$ hasta su análisis.

\section{Métodos}

Para realizar las mediciones de T4 total, TSH y Phe se emplearon los estuches de reactivos
UMELISA TSH Neonatal, UMELISA T4 Neonatal y el UMTEST PKU, respectivamente, producidos por el Centro de Inmunoensayo (21-24).

Los calibradores y controles empleados se prepararon a partir de sangre total humana ajustada a un valor de hematocrito de $55 \%$ y dispensados sobre papel de filtro Schleicher y Schuell 2992. Se emplearon discos de $5 \mathrm{~mm}$ de diámetro de los calibradores, controles y muestras.

EI UMELISA TSH Neonatal es un ensayo inmunoenzimático heterogéneo tipo sandwich, en el cual se utilizan tiras de ultramicroelisa $(10 \mu \mathrm{L}$ por pocillo) como fase sólida, revestidas previamente con anticuerpos anticadena $\beta$ de la TSH. Las muestras se eluyen con $70 \mu \mathrm{l}$ de conjugado anti $\alpha \mathrm{TSH} /$ fosfatasa alcalina en solución tampón tris-carnero; luego, se depositan en las tiras de reacción y, finalmente, se añade el sustrato fluorigénico (4-metil-umbeliferil-fosfato). La curva estándar se validó contra el patrón 80558 de la OMS y abarca un rango útil de 10-200 mUI/I, con un límite de detección de $2 \mathrm{mUI} / \mathrm{l}$. La evaluación analítica mostró una precisión intra e interensayo de 6,2 y $7,4 \%$, respectivamente. El porcentaje de recuperación obtenido al añadir la hormona fue de 98 a $105 \%$ y no se evidenciaron interferencias significativas con otras hormonas hipofisiarias.

EI UMELISA T4 Neonatal es un ensayo heterogéneo inmunoenzimático competitivo, en el cual se utilizan tiras de ultramicroelisa $(10 \mu \mathrm{l}$ por pocillo) como fase sólida, revestidas previamente con anticuerpos anti-T4. Las muestras se eluyen con $70 \mu \mathrm{l}$ de conjugado T4/fosfatasa alcalina en solución tampón barbiturato-ANS; luego, se depositan en las tiras de reacción y, finalmente, se añade el sustrato fluorigénico (4-metilumbeliferil-fosfato). La curva estándar se preparó por método gravimétrico y abarca un rango útil de 25-400 nmol/l, con un límite de detección de 11 $\mathrm{nmol} / \mathrm{I}$. La evaluación analítica mostró una precisión intra e interensayo de 6,9 y $9,1 \%$, respectivamente. La recuperación analítica obtenida fue mayor de $95 \%$ y no se evidenciaron interferencias significativas con otras sustancias estructuralmente relacionadas.

EI UMTEST PKU es una ultramicroprueba fluorescente basada en el método de McCaman y 
Robins. En esta prueba, la Phe presente en la muestra se eluye con $70 \mu \mathrm{l}$ de etanol a $70 \% \mathrm{y}$, luego, reacciona con la ninhidrina en condiciones óptimas de $\mathrm{pH}$ y temperatura, formando un complejo poco fluorescente. Con la adición de iones cobre, se produce la amplificación de la fluorescencia, aumentando su intensidad por la previa adición de L-leucil-L-alanina a la mezcla de reacción. La curva estándar se preparó por método gravimétrico y abarca un rango útil de 180-2880 $\mu \mathrm{mol} / \mathrm{l}$, con un límite de detección de $50 \mu \mathrm{mol} / \mathrm{l}$. La evaluación analítica mostró una precisión intra e interensayo de 3,8 y $5,2 \%$, respectivamente. El porcentaje de recuperación obtenido fue de 94 a $115 \%$.

La medición de la señal de fluorescencia se realizó en todos los casos, empleando el lector fluorímetro-fotómetro PR-521 y los resultados se calcularon automáticamente empleando el Strips reader software, versión7.0 para Windows, un programa diseñado para el cálculo de la concentración e interpretación de los resultados. Otros equipos y accesorios empleados fueron el lavador automático MAS 301 y el perforador P-51.

\section{Resultados y discusión}

De junio a septiembre de 1998, se analizaron 458 muestras de niños nacidos en el Hospital Docente Materno-Infantil 10 de Octubre en la Ciudad de La Habana.

En la figura 1 se muestra una distribución de frecuencias de los niveles de concentración de TSH en sangre del cordón umbilical en papel de filtro.

La media obtenida fue de $1,7 \mathrm{mU}$ de $\mathrm{TSH} / \mathrm{l}$ de sangre total $(E E M \pm 0,3 \mathrm{mUl} / \mathrm{l})$. En el cuadro $1 \mathrm{se}$ resume el comportamiento de los valores de TSH en la población estudiada de recién nacidos. El 98 y $99 \%$ de los recién nacidos tenían concentraciones de TSH inferiores a 8,6 y 10,3 $\mathrm{mUI} / \mathrm{l}$, respectivamente.

En la figura 2 aparece la distribución de valores de T4. La media de concentración obtenida fue de $177,2 \mathrm{nmol}$ de $T 4 / L$ de suero $(E E M \pm 0,02 \mathrm{nmol} / \mathrm{l})$.

El comportamiento de los valores de T4 en la población de recién nacidos estudiada se muestra en el cuadro 2.



Figura 1. Distribución de frecuencias de TSH en muestras de sangre de cordón umbilical en papel de filtro.

Cuadro 1. Concentraciones de TSH para el total de muestras evaluadas.

\begin{tabular}{lccc}
\hline Concentración (mUI/I) & $0-10$ & $10-20$ & $>20$ \\
Número de muestras & 446 & 8 & 1 \\
Porcentaje (\%) & 98 & 1,7 & 0,2 \\
\hline
\end{tabular}

El décimo percentil de la distribución correspondió a $116,4 \mathrm{nmol} / \mathrm{l}$.

Como resultado de este estudio, encontramos un niño cuya muestra al momento del análisis tenía valores de TSH y T4 de 74,6 $\mathrm{mUl} / \mathrm{l}$ y $40,1 \mathrm{nmol} / \mathrm{l}$, respectivamente, el cual se confirmó luego como hipotiroideo.

En la figura 3 se muestra la distribución de valores de Phe en muestras de sangre del cordón. La media fue de $41,6 \mu \mathrm{mol}$ de Phe/l de sangre total $(\mathrm{EEM} \pm 0,05 \mu \mathrm{mol} / \mathrm{l})$.

Las concentraciones de Phe en los de recién nacidos estudiados se presentan en el cuadro 3.

El 98 y $99 \%$ de la población estudiada tuvo valores inferiores a 81,4 y $84,8 \mu \mathrm{mol} / \mathrm{l}$, respectivamente.

Los resultados anteriores sugieren que es factible el empleo de muestras en sangre del cordón para el tamizaje de hipotiroidismo congénito. Es importante tener presente que en las primeras horas de vida ocurren cambios drásticos en el metabolismo de las hormonas tiroideas. La concentración de TSH en suero tiene un valor máximo a los 30 minutos después del nacimiento, 


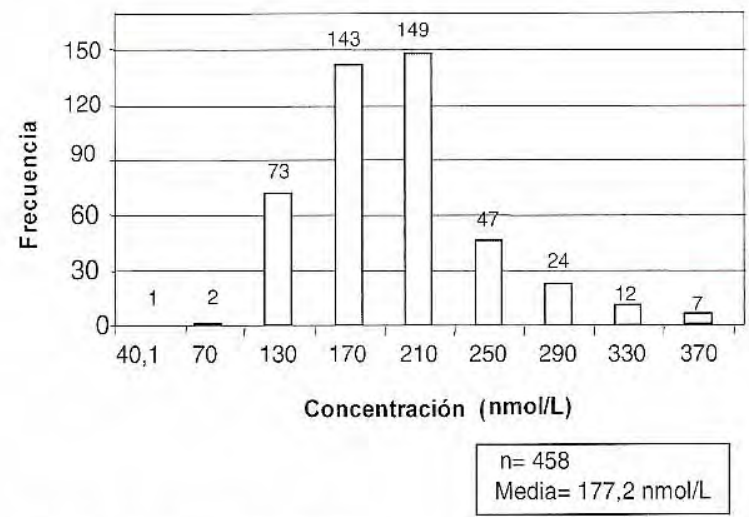

Figura 2. Distribución de frecuencias de T4 en muestras de sangre de cordón umbilical en papel de filtro.

Cuadro 2. Concentraciones de T4 para el total de muestras evaluadas.

\begin{tabular}{lccc}
\hline Concentración (nmol/l) & $<60$ & $60-116$ & $116-370$ \\
Número de muestras & 1 & 44 & 413 \\
Porcentaje (\%) & 0,2 & 9,6 & 90,2 \\
\hline
\end{tabular}

decrece rápidamente durante las primeras 24 horas de vida y más lentamente en los próximos 2 o 3 dias; y las concentraciones de T4 libre y total en suero tienen un valor máximo a las 240 36 horas después del parto y, luego, decrece lentamente durante las siguientes 2 o 3 semanas de vida $(25,26)$.

Los valores de estas hormonas en sangre del cordón no se comportan igual que en la sangre de talón e, incluso, en talón se modifican con la edad.

Los niveles de TSH y T4 de niños normales son más altos en sangre del cordón que en sangre tomada en los primeros días de vida. Por esta razón, es necesario establecer el nivel de corte adecuado para hacer un diagnóstico correcto y disminuir el número de casos reportados.

Al emplear un nivel de corte para TSH de $15 \mathrm{mUI} / \mathrm{l}$, el índice de reportados fue de 1\%; sin embargo, cuando se recalculó el nivel de corte para muestras de sangre del cordón, el percentil 97,5 de la distribución correspondió a 21,6 $\mathrm{mUI} / \mathrm{l}$ y con este nuevo valor, se bajó el porcentaje de falsos positivos a $0,5 \%$ sin encontrar ningún falso



Figura 3. Distribución de frecuencias de Phe en muestras de sangre de cordón umbilical en papel de filtro.

Cuadro 3. Concentraciones de Phe para el total de muestras evaluadas.

\begin{tabular}{lccc}
\hline Concentración $(\mu \mathrm{mol} / \mathrm{l})$ & $0-60$ & $60-120$ & $>120$ \\
Número de muestras & 367 & 88 & 2 \\
Porcentaje $(\%)$ & 80,3 & 19,2 & 0,4 \\
\hline
\end{tabular}

negativo (19). Se ha descrito que hay una tendencia a la disminución progresiva de los percentiles 98 y 99 para los valores de TSH en las muestras de sangre de talón tomadas a las 48 horas de 8,7 y $9,7 \mathrm{mUI} / \mathrm{I}$, respectivamente, y aquellas tomadas hasta los 14 días de 7,2 y 8,2 $\mathrm{mUI} / \mathrm{l}$, respectivamente (27). Mientras que Larsson y colaboradores reportaron una media para T4 de $142 \mathrm{nmol} / \mathrm{l}$ de plasma (EEM $\pm 1,7 \mathrm{nmol} / \mathrm{l}$ ), en una distribución de 19.289 muestras de sangre de talón de recién nacidos tomadas al quinto día de vida (28).

Así, podemos encontrar países como Chile, Costa Rica y Argentina que emplean un nivel de corte para TSH de $20 \mathrm{mUI} / /$ para muestras de sangre de talón entre el segundo y séptimo día de vida (29-31); en otros casos, como México, emplean $15 \mathrm{mUI} / \mathrm{l}$ para sangre de talón y $30 \mathrm{mUI} / \mathrm{l}$ para sangre del cordón umbilical (32,33); Cuba, $30 \mathrm{mUI} / \mathrm{l}$ para muestras de suero del cordón umbilical (17); España, 10 mUl/I para talón (34); EUA, 30 mUl/I para muestras tomadas antes de las 24 horas de vida y $25 \mathrm{mUI} / \mathrm{l}$ para aquellas tomadas a las 24 horas o más (35), y Japón, $8 \mathrm{mUI} / /$ para muestras de sangre de talón (36). 
Esta gran diversidad demuestra que, teniendo en cuenta los diferentes factores genéticos y ambientales que actúan sobre poblaciones de diferentes localizaciones geográficas, se recomienda que cada laboratorio establezca sus propios valores de referencia.

Para establecer un programa de tamizaje en sangre del cordón umbilical, es necesario tomar en cuenta la influencia de factores perinatales relacionados con el momento del parto y que pueden modificar las concentraciones de TSH y de las hormonas tiroideas en sangre del cordón (37); además, considerar el hecho de que el uso de antisépticos yodados también pueden interferir en los resultados porque la permeabilidad de la piel del niño genera una sobrecarga de yodo en la tiroides que puede generar bocio e hipotiroidismo (38).

El uso de la TSH como prueba de tamizaje se impone hoy en día en la mayoría de los programas de diagnóstico de hipotiroidismo congénito en el mundo por sus indiscutibles ventajas y la baja frecuencia de aparición de hipotiroidismo secundario y terciario (39). Nuestras observaciones sugieren que el uso de muestras de sangre de cordón umbilical para tamizaje primario, empleando $\mathrm{TSH}$, puede ser adecuado si se consideran criterios apropiados de la relación nivel de corte/casos recitados y, por tanto, un costobeneficio aceptable sobre la base de una buena eficiencia (40).

La concentración de Phe en el recién nacido puede ser normal hasta el cuarto día de vida, pero aumenta con rapidez al comenzar la alimentación proteica (41). Esta es la principal razón por la cual la mayoría de los programas de tamizaje de fenilcetonuria usan muestras de neonatos entre el quinto y el séptimo día de vida. Sin embargo, en la actualidad, el egreso temprano de las salas de maternidad ha llevado a una tendencia evidente de recolectar la muestra antes de las 48 horas de vida (42).

Vieira y Urquiza informaron una media para Phe de $98,2 \mu \mathrm{mol} / \mathrm{l}$, donde el 98 y el 99 percentil de una distribución de 42.917 muestras de sangre de talón de recién nacidos correspondió a 152 y $159 \mu \mathrm{mol} / \mathrm{l}(43)$.
Según los resultados obtenidos en nuestro estudio, los niveles de Phe en sangre del cordón son muy bajos y se corre el riesgo de que algunos niños que tienen deficiencia de la actividad del complejo enzimático hidroxilasa de fenilalanina, no tengan niveles elevados de fenilalanina en sangre en las primeras horas siguientes al nacimiento. De acuerdo con lo informado para muestras de sangre de talón, los valores elevados están por encima de $240 \mu \mathrm{mol} / \mathrm{l}$ ( $4 \mathrm{mg} / \mathrm{dl}$ ) y, sin embargo, nosotros obtuvimos solo dos valores mayores de 120 Hmol/l $(2 \mathrm{mg} / \mathrm{dl})$, pero muy cercanos a este valor. Esto indica que la concentración detectable de Phe en sangre del cordón es muy baja. A pesar de que en este estudio no detectamos ningún falso negativo, según lo informado en la literatura, si se usa un nivel de corte de $4 \mathrm{mg} / \mathrm{dl}$, se pueden perder de 70 a $80 \%$ de los niños con fenilcetonuria clásica (20). Dos niños con esta patología clásica mostraron los siguientes niveles de Phe: en sangre del cordón umbilical, 1,8 y $3,65 \mathrm{mg} / \mathrm{dl}$; a las 12 horas de edad, 4,2 y $5,3 \mathrm{mg} / \mathrm{dl}$; y a las 48 horas de vida, 5,6 y 6,6 $\mathrm{mg} / \mathrm{dl}$ (42).

Otros autores refieren que, a medida que aumenta la edad, aumentan los niveles de Phe y se puede usar un nivel de corte de $2 \mathrm{mg} / \mathrm{dl}$ para muestras de talón tomadas entre las 24 y 48 horas de vida $(42,44-47)$.

Así, podemos encontrar diferentes programas: en Argentina emplean un nivel de corte para PKU de $2 \mathrm{mg} / \mathrm{dl}$ para muestras de sangre de talón tomadas después de 48 horas de vida (45), 2,5 mg/dl para muestras de talón tomadas antes del alta (48) y 3 $\mathrm{mg} / \mathrm{dl}$ para muestras entre 36 y 96 horas de vida (49); en Uruguay, $3 \mathrm{mg} / \mathrm{dl}$ para muestras tomadas después de las 40 horas de vida (50); en Chile, 2 $\mathrm{mg} / \mathrm{dl}$ para muestras tomadas entre las 40 y 168 horas de vida (15), y en Costa Rica, 4 mg/dl para muestras de talón tomadas antes del egreso de la maternidad (30).

Los resultados obtenidos en nuestro estudio nos llevan a pensar que es inaceptable, por el momento, establecer un tamizaje para esta enfermedad antes de las 24 horas de vida, pues de algún modo estaríamos corriendo el riesgo de no detectar por tamizaje a los niños con esta dolencia. 


\section{Referencias}

1. Guthrie R, Susi A. A simple phenylalanine method for detecting phenylketonuria in large populations of newborn infants. Pediatrics 1963;32:338.

2. Dussault JH, Coulumbe P, Laberge C, Letarte J, Guyda H, Khoury R. Preliminary report on a mass screening program for neonatal hypothyroidism. J Pediatr 1975;86:670-4.

3. Klein AH, Augustine AV, Foley TP. Successful laboratory screening for congenital hypothyroidism. Lancet 1974;ii:77-9.

4. Lubenow N, Diepenbrock F, Schickling H, Bock D, Heckler R, Sander J. Phenylketonuria screening with a fluorometric microplate assay. Eur $\mathrm{J}$ Clin Chem Clin Biochem 1994;32:525-8.

5. Matalon R, Michals K. Phenylketonuria: screening, treatment and maternal PKU. Clin Biochem 1991;24:337.

6. Sanjurjo P. Efectos nutricionales secundarios de la dieta de PKU. En: Motger Catá J, editor. Prevención de enfermedades metabólicas congénitas. Madrid: Sociedad Española de Química Clínica; 1982. p.13.

7. KurtoÈlu S, Tutuê A, Aydin K, Genç E, Çaksen H. Persistent neonatal hypoglycemia: an unusual finding of congenital hypothyroidism. J Pediat Endocrinol Metabol 1998;11:277-9.

8. Dussault JH.The impact of systemic screening for congenital hypothyroidism. En: Farriaux JP, Dhondt JL, editors. New horizons in neonatal screening. Proceedings, $9^{\text {th }}$ International Screening Symposium, 1993, Sep. 13-17; Lille, Francia: International Society for Neonatal Screening; 1993. p.25-38.

9. Fisher DA. Screening for congenital hypothyroidism. TEM 1991;2:129-33.

10. Raiti S, Newns GA. Cretinism: early diagnosis and its relation to mental prognosis. Arch Dis Child 1971;46:692.

11. Klein AH, Meltzer S, Kenny FM. Improved diagnosis of congenital hypothyroidism treated before age 3 months. J Pediatr 1972;81:912.

12. Law WY, Bradley DM, Lazarus JH, John R, Gregory JW. Congenital hypothyroidism in Wales (1982-1993): demographic features, clinical presentation and effects on early neurodevelopment. Clin Endocrinol 1998;48:2017 .

13. Dugbartey AT. Neurocognitive aspects of hypothyroidism. Arch Intern Med 1998;158:1413-8.

14. Chiovato L, Giusti L, Tonacchera M, Ciampi M, Mammoli C, Lippi F, et al. Evaluation of L-thyroxine replacement therapy in children with congenital hypothyroidism. J Endocrinol Invest 1991;14:957-64.

15. Cornejo V. Programa de detección neonatal de errores innatos del metabolismo. Programa de búsqueda masiva de fenilcetonuria e hipotiroidismo en Chile. En: Colombo
M, Cornejo V, Raimann E, editores. Errores innatos del metabolismo del niño. Primeraedición. Santiago de Chile: Universitaria S.A.; 1999. p.314-9.

16. Iorcansky S. Hipotiroidismo congénito $(\mathrm{HC})$ en Latinoamérica. En: Cornejo V, Raimann E, Colombo M, editores. Resúmenes, II Congreso Latinoamericano de Errores Innatos del Metabolismo y Pesquisa Neonatal, 1999, 2427 de octubre. Santiago de Chile: Caupolicán Servicios Gráficos; 1999. p.30.

17. Carvajal F, Alvarez MA, Robaina R, Frómeta A, Fernández Yero JL, Guell R. Cuba: Programa Nacional de Diagnóstico Precoz de Hipotiroidismo Congénito, 198899. En: Cornejo V, Raimann E, Colombo M, editores. Resúmenes, II Congreso Latinoamericano de Errores Innatos del Metabolismo y Pesquisa Neonatal, 1999, 2427 de octubre. Santiago de Chile: Caupolicán Servicios Gráficos; 1999. p.61-2.

18. Aznarez A, Queiruga G, Frocht R, Laurani I, Cuesta V, Ruocco G. Uruguay: un programa nacional de pesquisaje neonatal de hipotiroidismo congénito unido al plan de inmunizaciones. En: Cornejo V, Raimann E, Colombo $M$, editores. Resúmenes, II Congreso Latinoamericano de Errores Innatos del Metabolismo y Pesquisa Neonatal, 1999, 24-27 de octubre. Santiago de Chile: Caupolicán Servicios Gráficos; 1999. p.46-7.

19. Prieto L, Gruñeiro de Papendieck L, Chiesa A, Bergadá C. Screening for congenital hypothyroidism $(\mathrm{CH})$ : experience in cord blood. En: Levy HL, Hermos RJ, Grady GF, editors. Proceedings, Third Meeting of the International Society for Neonatal Screening, 1996, Oct. 20-23. Boston: New England Regional Newborn Screening Program; 1996. p.271-2.

20. McCabe ERB, McCabe L, Mosher GA, Allen RJ, Berman JL. Newborn screening for phenylketonuria: predictive validity as a function of age. Pedi-atrics 1983;72:390-8.

21. Frómeta A, Lechuga MF, Pérez PL, Marrero $\mathbf{N}$, Urquiza HD, Coto R, et al. Desenvolvimento do UMELISA TSH neonatal para dosagem de TSH em sangue coletado em papel filtro. Rev Bras Anál Clín 1996;28:202-4.

22. Frómeta A, Lechuga MF, Pérez PL, Marrero N, Solís RL,Robaina R, et al. Development of an UMELISA TSH Neonatal for the quantification of thyroid stimulating hormone (TSH) in blood spot collected on filter paper. En: Levy HL, Hermos RJ, Grady GF, editors. Proceedings, Third meeting of the International Society for Neonatal Screening, 1996, Oct. 20-23. Boston: New England Regional Newborn Screening Program ;1996. p.238-9.

23. Almenares $\mathbf{P}$, Lechuga MF, Marrero $\mathbf{N}$, Solís $\mathbf{R L}$, Frómeta A, Robaina R, et al. Development of UMELISA T4 Neonatal for the early diagnosis of congenital hypothyroidism. En: Levy HL, Hermos RJ, Grady GF, editors. Proceedings, Third Meeting of the International Society for Neonatal Screening, 1996, Oct. 20-23. Boston: New England Regional Newborn Screening Program; 1996. p.240-1. 
24. Machado C, Solís RL, Bécquer D, Cazanave J, Fernández JL. The ultramicroanalytical system (SUMA) as a new approach to the newborn screening for hyperphenylalaninemias. En: Levy $\mathrm{HL}$, Hermos RJ, Grady GF, editors. Proceedings, Third Meeting of the International Society for Neonatal Screening, 1996, Oct. 20-23. Boston: New England Regional Newborn Screening Program; 1996. p.238-9.

25. Kaplan MM. Metabolism of thyroid hormones. En: Dekker $M$, editor. Basic and clinical endocrinology. New York: Marcel Dekker Inc.; 1983. p.11-35.

26. Ingbar SH, Woeber KA. La glándula tiroides. En: Williams RH, editor. Tratado de Endocrinología. Quinta edición. Madrid: Universidad de Madrid; 1984. p.129259.

27. Vieira Neto E, Urquiza HD. Distribución de la edad del recién nacido al momento de la toma de la muestra y su influencia en el punto de corte en un programa público de pesquisa neonatal de hipotiroidismo congénito en la ciudad de Rio de Janeiro. En: Cornejo V, Raimann E, Colombo $M$, editores. Resúmenes, II Congreso Latinoamericano de Errores Innatos del Metabolismo y Pesquisa Neonatal, 1999; 24-27 de octubre. Santiago de Chile: Caupolicán Servicios Gráficos; 1999. p.65.

28. Larsson A, Ljunggren JG, Ekman K, Nilsson A, Olin P. Screening for congenital hypothyroidism. Acta Paediatr Scand 1981;70:141-6.

29. Cuello X, Pérez P, Vivanco X, Lobo G, Pérez A, Bruggendieck B. Evaluación de 7 años de programa de detección precoz de hipotiroidismo congénito $(\mathrm{HC})$. En: Cornejo V, Raimann E, Colombo M, editores. Resúmenes, II Congreso Latinoamericano de Errores Innatos del Metabolismo y Pesquisa Neonatal, 1999, 24-27 de octubre. Santiago de Chile: Caupolicán Servicios Gráficos; 1999. p.54-5.

30. Casco T, Trejos R, Artavia E, Saborio M, Abarca G, Sánchez A, et al. Programa Nacional de Tamizaje Neonatal en Costa Rica. En: Cornejo V, Raimann E, Colombo $\mathrm{M}$, editores. Resúmenes, II Congreso Latinoamericano de Errores Innatos del Metabolismo y Pesquisa Neonatal, 1999, 24-27 de octubre. Santiago de Chile: Caupolicán Servicios Gráficos; 1999. p.59.

31. Barteik ME, Bonafé M, Conta AG, Santa María B, Tabares S, Moreno Barral J. Pesquisa neonatal para hipotiroidismo congénito y fenilcetonuria realizado por la universidad, en la provincia de Córdoba, Argentina. En: Cornejo V, Raimann E, Colombo M, editores. Resúmenes, II Congreso Latinoamericano de Errores Innatos del Metabolismo y Pesquisa Neonatal, 1999, 24-27 de octubre. Santiago de Chile: Caupolicán Servicios Gráficos, 1999. p.41-2.

32. Fernández GI, Benítez MG, Loza BD. Experiencia del sistema de vigilancia epidemiológica de hipotiroidismo congénito en el Instituto Mexicano del Seguro Social (IMSS). En: Cornejo V, Raimann E, Colombo M, editores. Resúmenes, II Congreso Latinoamericano de Errores Innatos del Metabolismo y Pesquisa Neonatal, 1999, 24-27 de octubre. Santiago de Chile: Caupolicán Servicios Gráficos; 1999. p.46.

33. Lemus H, Loza D, Benítez MG, Escobedo J, Fernández I, Zárate A. Congenital hypothyroidism newborn screening in Mexico. Abstracts, Fourth Meeting of the International Society for Neonatal Screening. 1999, june 13-16. Stockholm: Stockholm Convention Bureau; 1999. p.90.

34. Garriga MJ, Gil F, Luque I, Peran S. Criterios para establecer el nivel de corte de la TSH en el cribado del hipotiroidismo congénito. En: Cornejo $V$, Raimann $E$, Colombo $M$, editores. Resúmenes, II Congreso Latinoamericano de Errores Innatos del Metabolismo y Pesquisa Neonatal, 1999, 24-27 de octubre. Santiago de Chile: Caupolicán Servicios Gráficos; 1999. p.67-8.

35. Sardovia Iyer M, Hamilton B, Little Ch, Ayers L, Becker W, Lott J. Benefits of two-tier cut-off for TSH hypothyroidism assay. Abstracts, Fourth Meeting of the International Society for Neonatal Screening, 1999, june 13-16. Stockholm: Stockholm Convention Bureau; 1999. p.77.

36. Takahaschi M, Kitamura T, Imamura H, Miura M, Morijiri Y. Pitfalls of neonatal screening for congenital hypothyroidism. En: Levy HL, Hermos RJ, Grady GF, editors. Proceedings, Third Meeting of the International Society for Neonatal Screening, 1996, Oct. 20-23. Boston: New England Regional Newborn Screening Program; 1996. p.261-2.

37. Fuse Y, Wakae E, Nemoto Y, Uga N, Tanaka M, Maeda $\mathrm{M}$, et al. Influence of perinatal factors and sampling methods on TSH and thyroid hormone levels in cord blood. Endocrinol Japon 1991;38:297-302.

38. Po Lin C, Chen W, Wen Wu K. Povidone-iodine in umbilical cord care interferes with neonatal screening for hypothyroidism. Eur J Pediatr 1994;153:756-8.

39. Wang ST, Pizzolato S, Demshar HP. Diagnostic effectiveness of TSH screening and of T4 with secondary TSH screening for newborn congenital hypothyroidism. Clinica Chimica Acta. 1998;274:151-8.

40. Farriaux JP. Editorial. En: Farriaux JP, editor. La Depeche. Paris: Association francaise pour le dépistage et la prevention des handicaps de l'enfant; 1998. p.26.

41. Harrison TR. Trastornos del metabolismo. Trastornos hereditarios del metabolismo de los aminoácidos. En: Braunwald E, Isselbacher KJ, Peterdosdorf RG, Wilson JD, Martin JB, Fauci AS, editores. Principios de medicina interna. Séptima edición. México, D.F.: Interamerican McGraw-Hill; 1989. p.1967-72.

42. Cerone R, Caruso U, Schiaffino MC, Gazzolo A, Barella C, Romano C. Early newborn screening for phenylketonuria: phenylalanine levels during the first 24 hours of live. En: Levy HL, Hermos RJ, Grady GF, 
editors. Proceedings, Third Meeting of the International Society for Neonatal Screening, 1996, Oct. 20-23. Boston: New England Regional Newborn Screening Program; 1996. p.293-4.

43. Vieira Neto E, Urquiza HD. Valores normales de fenilalanina en muestras de sangre seca en papel de filtro de la pesquisa neonatal de la ciudad de Rio de Janeiro. En: Cornejo V, Raimann E, Colombo M, editores. Resúmenes, II Congreso Latinoamericano de Errores Innatos del Metabolismo y Pesquisa Neonatal, Santiago de Chile, 1999, 24-7 de octubre. Santiago de Chile: Caupolicán Servicios Gráficos; 1999. p.88.

44. Garriga MJ, Gil F, Luque I, Peran S. Criterios para establecer el nivel de corte de la TSH en el cribado del hipotiroidismo congénito. En: Cornejo V, Raimann E, Colombo $M$, editores. Resúmenes, II Congreso Latinoamericano de Errores Innatos del Metabolismo y Pesquisa Neonatal, 1999, 24-27 de octubre. Santiago de Chile: Caupolicán Servicios Gráficos; 1999. p.67-8.

45. Chiesa A, Gruñeiro de Papendieck L, Prieto L, Kesselman A, Goso S, Nadal MA, et al. Neonatal screening for PKU and hyperphenylalaninaemias (HPA): an experience in Argentina. En: Levy HL, Hermos RJ, Grady GF, editors. Proceedings, Third Meeting of the International Society for Neonatal Screening, 1996, Oct. 20-23. Boston: New England Regional Newborn Screening Program; 1996. p.291-2.

46. Carvalho TM, Costa MMR, Vargas P, Schmidt BJ. The effects of early discharge on PKU screening. En: Levy HL, Hermos RJ, Grady GF, editors. Proceedings, Third Meeting of the International Society for Neonatal Screening, 1996, Oct. 20-23: Boston: New
England Regional Newborn Screening Program; 1996. p.295-6

47. Dussault JH, Grenier A. L'importance d'evaluer le point de coupure du taux de TSH dans un programme de dépistage de l'hypothyroidie congénitale. En: Farriaux JP, editor. La Depeche. Paris: Bulletin de l'Association francaise pour le dépistage et la prevention des handicaps de l'enfant 1998;26.

48. Spécola N, Nuñez M, Balán A, Serodino S, Moretti A, Muschietti L, et al. Programa de screening de PKU en la provincia de Buenos Aires. En: Cornejo V, Raimann $E$, Colombo $M$, editores. Resúmenes, II Congreso Latinoamericano de Errores Innatos del Metabolismo y Pesquisa Neonatal, 1999, 24-27 de octubre. Santiago de Chile: Caupolicán Servicios Gráficos; 1999. p.55-6.

49. Narducci V, Sosa M, Lescano M, Lorenzo S, Boschetti N. Evolución del programa de pesquisa neonatal de hiperfenilalaninemias en el Hospital Nacional Profesor Dr. A. Posadas de Buenos Aires, Argentina En: Cornejo V, Raimann E, Colombo M, editores. Resúmenes, II Congreso Latinoamericano de errores innatos del metabolismo y pesquisa neonatal, 1999, 24 27 de octubre. Santiago de Chile: Caupolicán Servicios Gráficos; 1999. p.87.

50. Queiruga G, Machado ME, Nuñez S. Pesquisa de niños fenilcetonúricos en el banco de previsión social. Montevideo, Uruguay. En: Cornejo V, Raimann E, Colombo $\mathrm{M}$, editores. Resúmenes, II Congreso Latinoamericano de Errores Innatos del Metabolismo y Pesquisa Neonatal, 1999, 24-27 de octubre. Santiago de Chile: Caupolicán Servicios Gráficos; 1999. p.55. 